Stoyu Barov and Jan J. Dijkstra, Department of Mathematics, The University of Alabama, Box 870350, Tuscaloosa, Alabama 35487-0350.

e-mail: stoyu@ua.edu and jdijkstr@obelix.math.ua.edu

\title{
MORE ON COMPACTA WITH CONVEX PROJECTIONS
}

\begin{abstract}
We obtain information about the structure of nonconvex compacta $C$ in $\mathbb{R}^{n}(n \geq 3)$ having the property that every projection onto a hyperplane is convex. We find that if $\operatorname{dim}(C) \leq n-2$, then $C$ contains $n+1$ copies of $S^{n-2}$ that are contained in distinct hyperplanes. If $\operatorname{dim}(C) \geq n-1$, then the existence of three and no more than three hyperplanes that intersect $C$ in an $(n-2)$-sphere can be guaranteed.
\end{abstract}

Consider the vector space $\mathbb{R}^{n}$ for $n \geq 3$. Let us call the image of a set $X \subset \mathbb{R}^{n}$ under an orthogonal projection onto a hyperplane a shadow of $X$. Borsuk [1] has shown that there exist Cantor sets in $\mathbb{R}^{n}$ such that all their shadows are $(n-1)$-dimensional. The projections of such Cantor sets contain $(n-1)$ dimensional convex bodies but the following result shows that these shadows must be 'frayed around the edges'. According to Cobb [2] every compactum $C$ in $\mathbb{R}^{n}$ with the property that all its shadows are convex bodies contains an arc. Dijkstra, Goodsell, and Wright [3] improved on this result by showing that such a $C$ must contain an $(n-2)$-sphere; so in this case projections cannot raise dimension by more than one. The estimate that $\operatorname{dim}(C) \geq n-2$ was also shown to be sharp.

Our aim in this paper is to obtain precise information about the number of 'essentially distinct' $(n-2)$-spheres that can be found in these sets:

Theorem 1. If $C$ is a nonconvex compactum in $\mathbb{R}^{n}$ such that all its shadows are convex, then

(1) C contains an $(n-1)$-cell or

(2) there exist $n+1$ distinct hyperplanes $H_{0}, \ldots, H_{n}$ such that every $C \cap H_{i}$ contains an $(n-2)$-sphere.

Key Words: convex set, compact set, projection, hyperplane, shadow, facet

Mathematical Reviews subject classification: 46A55, 57N15

Received by the editors February 2, 2000 
Since every $(n-1)$-cell contains a continuum of disjoint $(n-2)$-spheres, a natural question is whether option (1) is necessary. We prove that (1) is essential by showing that without it the existence of three and no more than three hyperplanes that intersect $C$ in an $(n-2)$-sphere can be guaranteed.

Definitions. Consider a Cartesian coordinate system in the vector space $\mathbb{R}^{n}$ with origin $\mathbf{0}$. Also, we have the usual norm in $\mathbb{R}^{n}$ : if $x=\left(x_{1}, x_{2}, \ldots, x_{n}\right) \in$ $\mathbb{R}^{n}$, then $\|x\|=\sqrt{x_{1}^{2}+\cdots+x_{n}^{2}}$. $S^{n}$ stands for the standard sphere $\{x \in$ $\left.\mathbb{R}^{n+1}:\|x\|=1\right\}$ and an $n$-sphere is any space that is homeomorphic to $S^{n}$. An $n$-cell is a space that is homeomorphic to $\left\{x \in \mathbb{R}^{n}:\|x\| \leq 1\right\}$. A $k$-plane in $\mathbb{R}^{n}$ is a $k$-dimensional affine subspace and a hyperplane is an $(n-1)$-plane.

The boundary and interior of a subset $A$ of a topological space $X$ are denoted by $\partial_{X} A$ respectively $\operatorname{int}_{X} A$. If the ambient space is $\mathbb{R}^{n}$, then we use $\partial A$ and int $A$. We denote the convex hull of a set $C \subset \mathbb{R}^{n}$ by $\langle C\rangle$. A subset $B$ of $\mathbb{R}^{n}$ is called a convex body if $B$ is an $n$-dimensional convex compactum. So a convex body $B$ is an $n$-cell, it has non-empty interior in $\mathbb{R}^{n}$ and its boundary $\partial B$ is an $(n-1)$-sphere.

If $B$ is a convex body in $\mathbb{R}^{n}$, then a subset $F$ of $\partial B$ is called a facet of $B$ (or of $\partial B$ ) if there is a hyperplane $H$ such that $F=H \cap \partial B=H \cap B$ and $F$ is an $(n-1)$-cell. Note that the facets of $\partial B$ can meet only in their boundary points. We define the following compact subset of $\partial B$.

$$
\mathcal{E}(B)=\partial B \backslash \bigcup\left\{\operatorname{int}_{\partial B} F: F \text { is a facet of } B\right\} .
$$

The points of $\mathcal{E}(B)$ are extremal points with respect to projections onto hyperplanes.

Lemma 2. If $B$ is a convex body in $\mathbb{R}^{n}$ and $C$ is a compact subset of $\mathbb{R}^{n}$ that has the same shadows as $B$ in all directions, then $\mathcal{E}(B) \subset C \subset B$.

This lemma is a special case of Theorem 4.7 in [3]. We include a direct proof to display the geometry of the situation which is important in the sequel.

Proof. Let $B$ and $C$ be compacta in $\mathbb{R}^{n}$ such that $B$ is a convex body and $B$ and $C$ have identical projections. Note that according to Hahn-Banach (see e.g. [5, Theorem 3.4]) the convex hull of $C$ is the intersection of all half spaces that contain $C$. Consequently, we have $\langle C\rangle=B$.

Assume that $x$ is some element of $\mathcal{E}(B) \backslash C$. Since $x$ is a point on the boundary of the convex compactum $B$, according to Hahn-Banach we can find a hyperplane $H$ through $x$ such that $B$ is contained in one of its closed half spaces, say $P$. Let $\ell$ be a line in $H$ through $x$ such that $x \notin \operatorname{int}_{\ell}(\ell \cap \partial B)$. Such a line can be found because if there are $n-1$ independent lines without 
this property, then $x$ must lie in the interior of the $(n-1)$-cell $H \cap \partial B$, which contradicts $x \in \mathcal{E}(B)$. Select a point in the interior of $B$ and let $M$ be the 2dimensional plane that contains the point and the line $\ell$. Select a coordinate system for $M$ such that the half plane $M \cap P$ corresponds with directions $[0, \pi]$ and $x$ is the pole. Noting that $x \notin C$ we let $\theta: M \cap C \rightarrow[0, \pi]$ be the continuous map that assigns to each point of the compactum its polar angle. If the range of $\theta$ contains both 0 and $\pi$, then $x \in \operatorname{int}_{\ell}(\ell \cap S)$, a contradiction. So we may conclude that $\theta$ is not surjective. Then by compactness of the range of $\theta$ we have that there is some $\alpha \in(0, \pi) \backslash \theta(M \cap C)$. Let $\ell^{\prime}$ be the line in $M$ through $x$ with direction $\alpha$. Then $\ell^{\prime}$ misses $C$. Let $p$ be the projection of $\mathbb{R}^{n}$ onto a hyperplane that is perpendicular to $\ell^{\prime}$. Note that $p(x) \notin p(C)$ but that $p(x) \in p(B)=p(\langle C\rangle)=\langle p(C)\rangle$. Consequently, $p(C)=p(B)$ is not convex and we have arrived at a contradiction. Hence $\mathcal{E}(B) \subset C$.

We now prove Theorem 1 .

Proof. Let $C$ be a nonconvex compactum in $\mathbb{R}^{n}$ such that the projection of $C$ in any direction is convex. Then $B=\langle C\rangle$ is a convex body because if $\operatorname{dim}(B)<n$, then by convexity $B$ is contained in a hyperplane and hence $C$ projects onto itself. We have $p(B)=p(\langle C\rangle)=\langle p(C)\rangle=p(C)$ for every projection $p$. Note that the boundary in $\partial B$ of each facet of $B$ is an $(n-2)$ sphere that is contained in $\mathcal{E}(B)$ and hence in $C$, by Lemma 2. So if there are more than $n$ facets we are finished. Assume that there are no more than $n$ facets. Then the union of all the facets $K$ is a compact set that does not cover the entire $\partial B$ because an $n$-cell in $\mathbb{R}^{n}$ cannot be bounded by only $n$ hyperplanes. So $\partial B \backslash K$ is a nonempty open subset of the $(n-1)$-sphere $\partial B$ that is contained in $\mathcal{E}(B)$ and hence in $C$. So $C$ contains an $(n-1)$-cell.

Example 1. We shall use [3, Theorem 5.3] which shows that for every convex body $B$ there is a zero-dimensional set $Z$ in the interior of $B$ such that $C=$ $Z \cup \mathcal{E}(B)$ is compact and has the same shadows as $B$. Consider an $n$-simplex $\Delta^{n}$ in $\mathbb{R}^{n}$. Its $n+1$ facets are the $(n-1)$-faces and $\mathcal{E}\left(\Delta^{n}\right)$ is the $(n-2)$-skeleton $\Delta_{n-2}^{n}$. Select a 0 -dimensional set $Z$ such that $C=\Delta_{n-2}^{n} \cup Z$ is a compactum with the same projections as $\Delta^{n}$. Note that $C$ is a 'minimal' example of a nonconvex compactum with convex shadows and that there are precisely $n+1$ hyperplanes $H$ such that $H \cap C$ contains an $(n-2)$-sphere.

Theorem 3. If $C$ is a nonconvex compactum in $\mathbb{R}^{n}$ with only convex shadows, then there exist at least three distinct hyperplanes whose intersection with $C$ contains an $(n-2)$-sphere. 
Proof. Put $B=\langle C\rangle$ and note that as in the proof of Theorem $1 B$ is a convex body and that $\mathcal{E}(B) \subset C$. Let

$$
\mathcal{H}=\{H: H \text { is a hyperplane that intersects } B \text { in a facet }\}
$$

If $|\mathcal{H}| \geq 3$, then we are done.

We show that if $|\mathcal{H}|<3$, then there are infinitely many hyperplanes whose intersection with $C$ contains an $(n-2)$-sphere. Let $x$ be an arbitrary point in the interior of $B$ and note that for any hyperplane $P$ that passes through $x$ the intersection $P \cap \partial B$ is an $(n-2)$-sphere. We consider three cases:

(1) $\mathcal{H}=\emptyset$. Then $\partial B=\mathcal{E}(B) \subset C$ so $C$ contains the $(n-2)$-sphere $P \cap \partial B$ for every hyperplane $P$ through $x$.

(2) $\mathcal{H}=\left\{H_{1}, H_{2}\right\}$ with $H_{1}$ and $H_{2}$ parallel or identical. Recall that $\partial B \subset$ $H_{1} \cup H_{2} \cup \mathcal{E}(B)$. If $P$ is the hyperplane through $x$ that is parallel to $H_{1}$ and $H_{2}$, then $P \cap \partial B=P \cap \mathcal{E}(B) \subset P \cap C$.

(3) $\mathcal{H}=\left\{H_{1}, H_{2}\right\}$ with $H_{1}$ and $H_{2}$ distinct and not parallel. Consider the ( $n-2)$-plane $L=H_{1} \cap H_{2}$. Let $P$ be the hyperplane that contains both $L$ and $x$. Note that $L$ cannot intersect the interiors of the facets so $P \cap \partial B=P \cap \mathcal{E}(B) \subset P \cap C$.

The proof is complete.

Theorem 4. For every $n \geq 3$ there exists a nonconvex compactum $C$ in $\mathbb{R}^{n}$ such that every shadow of $C$ is convex and with the property that there are precisely three distinct hyperplanes whose intersection with $C$ contains an $(n-2)$-sphere.

Proof. We define the hyperplanes

$$
\begin{aligned}
& H_{0}=\left\{x \in \mathbb{R}^{n}: x_{n}=0\right\} \\
& H_{1}=\left\{x \in \mathbb{R}^{n}: x_{1}=0\right\} \\
& H_{2}=\left\{x \in \mathbb{R}^{n}: x_{2}=0\right\}
\end{aligned}
$$

and the $(n-2)$-planes $Z_{0}=H_{1} \cap H_{2}, Z_{1}=H_{2} \cap H_{0}$, and $Z_{2}=H_{0} \cap H_{1}$. Let $F_{0}$ be one quadrant of the unit ball in $H_{0}$.

$$
F_{0}=\left\{x \in H_{0}: x_{1}, x_{2} \geq 0 \text { and }\|x\| \leq 1\right\},
$$

and consider the point $p=(0, \ldots, 0,1) \in \mathbb{R}^{n}$. If $A$ is a subset of $H_{0}$, then the cone over $A$ with respect to $p$ is defined by

$$
\mathfrak{C}(A)=\{t a+(1-t) p: t \in[0,1] \text { and } a \in A\}
$$


Observe that if $A \subset H_{0}$ is compact, then $\partial \mathfrak{C}(A)=A \cup \mathfrak{C}\left(\partial_{H_{0}} A\right)$.

The convex body $B$ is $\mathfrak{C}\left(F_{0}\right)$. Note that $F_{0}$ is a facet of $B$. The boundary of $F_{0}$ in $H_{0}$ consists of three $(n-2)$-cells,

$$
\begin{aligned}
& K_{1}=\left\{x \in Z_{1}: x_{1} \geq 0 \text { and }\|x\| \leq 1\right\}=Z_{1} \cap B, \\
& K_{2}=\left\{x \in Z_{2}: x_{2} \geq 0 \text { and }\|x\| \leq 1\right\}=Z_{2} \cap B, \\
& P^{*}=\left\{x \in H_{0}: x_{1}, x_{2} \geq 0 \text { and }\|x\|=1\right\} .
\end{aligned}
$$

Observe that for $i=1,2, K_{i}$ is a facet of $F_{0}$ in $H_{0}$ and hence

$$
F_{i}=\mathfrak{C}\left(K_{3-i}\right)=H_{i} \cap B
$$

is a facet of $B$. Let $P$ be the $(n-1)$-cell $\mathfrak{C}\left(P^{*}\right)$ and note that since $\partial_{H_{0}} F_{0}=$ $K_{1} \cup K_{2} \cup P^{*}$, we have that $\partial B=F_{0} \cup F_{1} \cup F_{2} \cup P$. Let $K_{0}$ stand for the $(n-2)$-cell,

$$
K_{0}=\mathfrak{C}\left(K_{1} \cap K_{2}\right)=Z_{0} \cap B .
$$

Observe that for $i=1,2, \partial_{Z_{i}} K_{i}=\left(K_{1} \cap K_{2}\right) \cup\left(K_{i} \cap P^{*}\right)$ and hence that

$$
\partial_{H_{i}} F_{i}=K_{3-i} \cup K_{0} \cup \mathfrak{C}\left(K_{3-i} \cap P^{*}\right) \subset K_{3-i} \cup K_{0} \cup P .
$$

So we may conclude that $C^{*}=K_{0} \cup K_{1} \cup K_{2} \cup P$ equals the boundary of $B$ with the interiors of the three facets $F_{0}, F_{1}$, and $F_{2}$ removed and hence that $\mathcal{E}(B) \subset C^{*}$.

We may now apply [3, Theorem 5.3] to obtain a zero-dimensional space $Z$ in the interior of $B$ such that $C=Z \cup C^{*}$ is compact and has the same projections onto hyperplanes as $B$. This completes the construction of the counterexample $C$.

Let $H$ be a hyperplane such that

$$
H \cap C \text { contains an }(n-2) \text {-sphere } S .
$$

We need to show that $H$ is one of the hyperplanes $H_{0}, H_{1}$, or $H_{2}$.

Remark. We shall use the Brouwer Invariance of Domain Theorem (see e.g. [4, Theorem 4.6.7]) and corollaries: every continuous injection of $\mathbb{R}^{n}$ into itself is an open mapping, no $n$-sphere can be embedded into $\mathbb{R}^{n}$, and no proper subspace of an $n$-sphere is an $n$-sphere.

Claim 1. $M=H \cap P$ does not contain $S$.

Proof. We consider two cases: 
(1) Let $p \notin H$. Then we can project $M$ onto the base $P^{*}$ of the cone $P$ via a continuous map $f: M \rightarrow P^{*}$ defined as follows: if $x \in M$, then $f(x)$ is the intersection point of $H_{0}$ and the line passing through $p$ and $x$. It is easy to see that $f$ is one-to-one (because $p \notin H$ ) and that $f$ is an embedding by the compactness of $M$. Since $P^{*}$ is an $(n-2)$-cell, according to Brouwer we have that $M$ cannot contain an $(n-2)$-sphere.

(2) Let $p \in H$. Then $M=\{p\}$ and we are finished or $M=\mathfrak{C}\left(M \cap P^{*}\right)$ and $L=H \cap H_{0}$ is a hyperplane in $H_{0}$. Note that $P^{*}$ is subspace of the standard unit sphere $S^{n-2}$ in $H_{0}$. The intersection $A$ of $S^{n-2}$ with the hyperplane $L$ is either a point or an $(n-3)$-sphere. If $A$ is a singleton, then $M$ is contained in the line segment $\mathfrak{C}(A)$ and if $A$ is an $(n-3)$ sphere, then $M$ is contained in the $(n-2)$-cell $\mathfrak{C}(A)$. Either way, by the Remark $M$ cannot contain an $(n-2)$-sphere $(n \geq 3)$.

Claim 2. $H \cap C^{*}=S$ and $C^{*}=\mathcal{E}(B)$.

Proof. Note that $S \backslash C^{*}$ is an open subset of the sphere $S$ and hence either empty or $(n-2)$-dimensional. Since $S \backslash C^{*} \subset Z$ and $Z$ is zero-dimensional, we know that $S$ is contained in $H \cap C^{*}$. We consider two cases:

(1) $H \cap$ int $B \neq \emptyset$. Then $H \cap \partial B$ is an $(n-2)$-sphere. Since $C^{*} \subset \partial B$, we have $S \subset H \cap C^{*} \subset H \cap \partial B$. According to the Remark $S=H \cap \partial B=H \cap C^{*}$.

(2) $H \cap$ int $B=\emptyset$. Then $H \cap \partial B=H \cap B$ is a convex $k$-cell $(k \leq n-1)$ which contains the $(n-2)$-sphere $S$. So by the Remark we have that $k=n-1$ and $H \cap \partial B$ is a facet of $B$. If $H \cap \partial B$ is facet other than $F_{0}, F_{1}$, or $F_{2}$, then it must be contained in $P$. Since $S \subset H \cap C^{*} \subset H \cap \partial B \subset H \cap P$, this violates Claim 1 . So $F_{0}, F_{1}$, and $F_{2}$ are the only facets of $B$ which means that $C^{*}=\mathcal{E}(B)$. Let $H \cap \partial B=F_{i}$. Then $H \cap C^{*}=\partial_{H_{i}} F_{i}$ is an $(n-2)$-sphere and hence $S=H \cap C^{*}$, by Brouwer.

Claim 3. $H \in\left\{H_{0}, H_{1}, H_{2}\right\}$.

Proof. Consider the set $L=S \backslash P=H \cap C^{*} \backslash P$ and note that it is a nonempty open subset of $S$ and therefore $(n-2)$-dimensional. We have $L \subset$ $K_{0} \cup K_{1} \cup K_{2} \subset Z_{0} \cup Z_{1} \cup Z_{2}$ so $\operatorname{dim}\left(L \cap Z_{i}\right)=n-2$ for some $i$. Consequently, $\operatorname{dim}\left(H \cap Z_{i}\right)=n-2$ so $H$ must contain the $(n-2)$-plane $Z_{i}$ and hence the origin $\mathbf{0}$. Since $\mathbf{0} \in C^{*} \backslash P$, we have $\mathbf{0} \in L$ where it has neighborhoods 
homeomorphic to $\mathbb{R}^{n-2}$. We want to show that $L$ is not a subset of the $(n-2)$ plane $Z_{i}$. Suppose $L \subset Z_{i}$. On the one hand we have according to Brouwer Invariance of Domain that $\mathbf{0}$ is an interior point of $L$ in $Z_{i}$. On the other hand, $\mathbf{0}$ is a boundary point in $Z_{i}$ of $K_{i}=Z_{i} \cap B$ and hence of its subset $L$, a contradiction.

Consequently, $L \backslash Z_{i}$ is a nonempty open subset of $S$ and hence $\operatorname{dim}\left(L \backslash Z_{i}\right)=$ $n-2$. By the same argument as above we find a $j \neq i$ such that $Z_{j} \subset H$. Since $H$ contains $Z_{i}$ and $Z_{j}$, we may conclude that $H$ equals $H_{k}$ where $k$ is the third index.

The proof of Theorem 4 is complete.

Example 2. For every open $O \subset \mathbb{R}^{n}$ we construct a closed, zero-dimensional subset of $O$ that has the same shadows as $O$. Putting $O=\mathbb{R}^{n}$ we see that Theorems 1 and 3 are no longer valid if we replace $C$ is compact by $C$ is closed.

Consider an open subset $O$ of $\mathbb{R}^{n}$ and choose a locally finite open cover $\left\{U_{i}: i \in \mathbb{N}\right\}$ for $O$ consisting of sets with compact closures in $O$. Shrink this cover to a closed cover $\left\{V_{i}: i \in \mathbb{N}\right\}$ with $V_{i} \subset U_{i}$ for every $i \in \mathbb{N}$. According to [1] or [3, Proposition 3.1] we can find for each $i \in \mathbb{N}$ a Cantor set $C_{i} \subset U_{i}$ such that every line in $\mathbb{R}^{n}$ that intersects $V_{i}$ also meets $C_{i}$. Put $C=\bigcup_{i=1}^{\infty} C_{i}$. Then, since $\left\{C_{i} \mid i \in \mathbb{N}\right\}$ is a locally finite collection of Cantor sets, we have that $C$ is closed in $O$ and zero-dimensional. Moreover, the shadows of $O$ and $C$ are identical.

Example 3. We show that the compactness of $C$ is also essential in Lemma 2. Consider $\mathbb{R}^{2}$ and let $S=\left\langle K_{1} \cup K_{2}\right\rangle$, where

$$
K_{1}=\left\{(x, y):(x+1)^{2}+y^{2}=1\right\}
$$

and

$$
K_{2}=\left\{(x, y):(x-1)^{2}+y^{2}=1\right\}
$$

Observe that if $\ell$ is a nonhorizontal line in $\mathbb{R}^{2}$ passing through the point $a=(1,1)$, then $\ell$ intersects the interior of $S$, see figure. If $\ell$ is the horizontal line through $a$, then $\ell \cap S=[-1,1] \times\{1\}=F$. Either way, we have that for every line $\ell$ through $a$ and every neighborhood $U$ of $a$ in $\ell$, the intersection $U \cap S \backslash\{a\}$ is nonempty.

Now, for $n \geq 3$ let $K$ be a convex body in $\mathbb{R}^{n-2}$ and define the convex body $B=S \times K \subset \mathbb{R}^{n}$ and its subset $C=B \backslash(\{a\} \times \operatorname{int} K)$. Note that the points in $B \backslash C$ are boundary points of the facet $F \times K$ of $B$. Consequently, we have $B \backslash C \subset \mathcal{E}(B)$.

We will show that every line that intersects $B \backslash C$ also intersects $C$, proving that $C$ and $B$ have identical shadows in every direction. Let $\pi: \mathbb{R}^{n} \rightarrow \mathbb{R}^{2}$ 


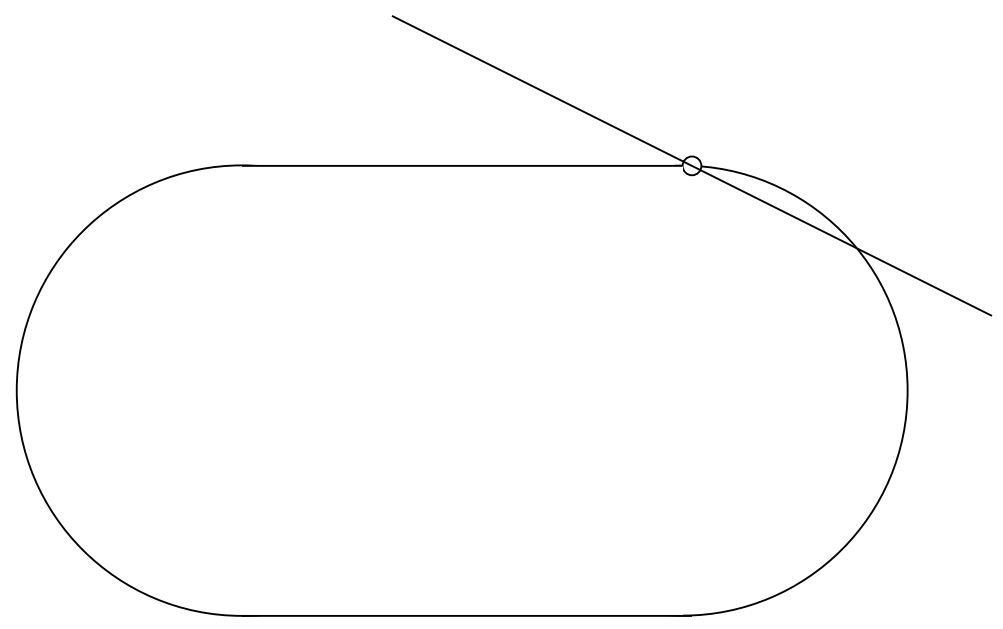

be the projection onto the first two coordinates. Let $\ell$ be a line in $\mathbb{R}^{n}$ that intersects $B \backslash C$ in a point $x=(a, b)$.

If $\pi(\ell)=\{a\}$, then we may treat $\ell$ as a line in $\mathbb{R}^{n-2}$. Since $\ell$ intersects the interior of $K$ (in $b$ ), we have that $\ell$ contains a boundary point of $K$, say $c$. Then $(a, c) \in \ell \cap C$.

If $\pi(\ell)$ is a line in the plane, then $U=\pi\left(\ell \cap\left(\mathbb{R}^{2} \times \operatorname{int} K\right)\right)$ is a neighborhood of $a$ in $\pi(\ell)$ and hence there is a $c \in U \cap S \backslash\{a\}$. Let $d \in \operatorname{int} K$ be such that $(c, d) \in \ell$. Note that $(c, d) \in \ell \cap C$.

\section{References}

[1] K. Borsuk, An example of a simple arc in space whose projections in every plane has interior points, Fund. Math. 34 (1947), 272-277.

[2] J. Cobb, Raising dimension under all projections, Fund. Math. 144 (1994), 119-128.

[3] J. J. Dijkstra, T. L. Goodsell, and D. G. Wright, On compacta with convex projections, Topology Appl. 94 (1999), 67-74.

[4] J. van Mill, Infinite-Dimensional Topology, Prerequisites and Introduction, North-Holland, Amsterdam, 1989.

[5] W. Rudin, Functional Analysis, McGraw-Hill, New York, 1973. 